\title{
Lange, Bastian; Hülz, Martina; Schmid, Benedikt; Schulz, Christian (Hrsg.) (2020): Postwachstumsgeographien. Raumbezüge diverser und alternativer Ökonomien.
}

\author{
Tanja Mölders
}

Eingegangen: 2. November 2020 - Angenommen: 2. Dezember 2020 - Online veröffentlicht: 22. Januar 2021

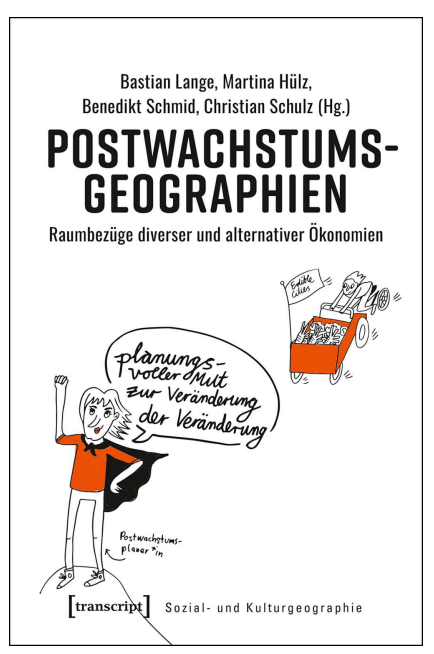

Mit ihrem Sammelband „Postwachstumsgeographien“ haben Bastian Lange, Martina Hülz, Benedikt Schmid und Christian Schulz eine theoretisch sowie empirisch äußerst fundierte Anthologie vorgelegt, in der die Schnittstelle zwischen raumwissenschaftlichen Zugängen und Konzepten und alternativen, das heißt primär nicht kapitalistisch orientierten, Ökonomien nicht nur ausgeleuchtet, sondern zugleich als eigenes raum- und planungswissenschaftliches Forschungs- und Handlungsfeld ausgearbeitet wird. Damit lässt sich der Band im noch jungen Themenfeld der raumbezogenen Nachhaltigkeitstransformation verorten

$\triangle$ apl. Prof. Dr. Tanja Mölders, Leibniz Universität Hannover, Hannover

t.moelders@archland.uni-hannover.de

(c) (1) (2) ( 2021 Mölders; licensee oekom verlag. This Open Access article is published under the Creative Commons Attribution-ShareAlike 4.0 International Licence. und eröffnet Wissenschaftlerinnen und Wissenschaftlern ebenso wie Praktikerinnen und Praktikern, aber auch Studierenden einen bisher nicht vorhandenen Überblick über raum- und planungswissenschaftliche Positionen im Kontext wachstumskritischer und heterodoxer Ökonomien. Dass der äußerst ansprechend gestaltete Band im transcript Verlag als E-Book kostenlos zur Verfügung steht, ist angesichts des Themas nur konsequent und dennoch hervorzuheben.

Die Anthologie ist in die vier Teile „Perspektivräume“, „Möglichkeitsräume“, „Konflikträume“ und „Gestaltungsräume“ gegliedert. In jedem dieser Räume sind sowohl theoretisch-konzeptionelle als auch empirisch-exemplarische Beiträge versammelt. Außerdem bereichern Interviews mit Praxisakteuren, die vielfach auch wissenschaftlich tätig sind, die einzelnen Teile und illustrieren die Erfahrungen transformativer Praxis. Besonders hervorzuheben ist die Einleitung, in der Herausgeber und Herausgeberin eine fundierte Verortung innerhalb der für die Thematik relevanten Diskursstränge (Nachhaltige Entwicklung, Postwachstum sowie raumbezogene Konzepte) leisten und auch sehr präzise die mit dem Band verfolgten Ziele definieren: Ihr Anliegen ist es, deutlich zu machen, inwiefern räumliche Perspektiven die Erkenntnisse über und Gestaltungsmöglichkeiten von Postwachstumsprozessen zu erweitern vermögen. Die von ihnen angelegten Verknüpfungen von raumbezogenen Konzepten, Zuschreibungen und Maßnahmen werden von den über 40 Autorinnen und Autoren der Einzelbeiträge aufgenommen, vertieft sowie auch differenziert.

Dabei vergrößern die Beiträge im Teil „Perspektivräume" die Blickwinkel, indem etwa die Kategorie Arbeit in einen räumlich gefassten Postwachstumskontext gestellt wird oder die Räumlichkeit von Postwachstumstransforma- 
tion entlang verschiedener Raumkonzepte ausbuchstabiert wird.

Der Fokus im Teil „Möglichkeitsräume“ liegt auf der Erkundung von mehr oder weniger etablierten Praktiken des Postwachstums. Räumliche Bezüge werden dabei nicht nur darüber hergestellt, dass diese Praktiken an konkreten Orten stattfinden, sondern auch über raumtheoretische Reflexionen (z. B. zu Konzepten von third space und third place). Auch wird die Rolle der (Stadt-)Planung in Postwachstumsprozessen adressiert und die Rolle ländlicher Räume in den Debatten um Postwachstum herausgearbeitet.

Die Beiträge im Teil „Konflikträume“ stellen Ambivalenzen der Postwachstumsdebatte dar. Die hier versammelten Überlegungen beispielsweise zur Dominanz eurozentristischer Perspektiven oder zu ablehnenden Haltungen gegenüber Postwachstumstransformationen verdeutlichen die Komplexität des Themenfeldes. Dass Spannungsfelder wie rechte Raumsemantiken auch in (Praxis-)Beiträgen anderer Teile des Bandes angesprochen werden, spricht für die mit der Anthologie insgesamt verfolgte Reflexivität.

Schließlich fragen die Beiträge im Teil „Gestaltungsräume“ danach, wie und durch wen räumliche Transformation in Richtung Nachhaltigkeit und Postwachstum möglich gemacht werden kann. Dabei geht es zunächst um grundsätzliche Auseinandersetzungen mit Begriffen und Konzepten von Transformation sowie unterschiedlichen Positionen in der Wachstumsdebatte. Damit wird nicht nur ein Beitrag zur Begriffsschärfung geleistet, sondern es werden auch Vorschläge für neue Konzepte (z. B. Vorsorgeorientierte Postwachstumsökonomie) erarbeitet. Andere Beiträge fokussieren die Akteure von Postwachstumstransformationen und setzen sich dabei dezidiert mit dem Rollenverständnis von Planerinnen und Planern sowie dem Bauhaus als Transformationsgestalter in Ostdeutschland auseinander.

Insgesamt bestätigen die versammelten Beiträge die in der Einleitung formulierte Annahme, dass Fragen nach den „Grenzen des Wachstums“ raum- und planungswissenschaftliche Fragen per se sind. ,Raum“ dient dabei erstens als Analyse- und Gestaltungskategorie sowie zweitens als inter- und transdisziplinäre Vermittlungskategorie. Dabei wird je nach disziplinärer Perspektive, thematischem Fokus und/oder Praxiserfahrungen deutlich, dass und wie die Raum- und Planungswissenschaften von Postwachstumsdebatten lernen und an diese anschließen können sowie umgekehrt raumbezogene Perspektiven Bereicherungen für Postwachstumsdebatten darstellen können. Es ist mit Blick auf einen Band mit dem Titel ,Postwachstumsgeographien" folgerichtig, dass dieser wechselseitige Lernprozess hier konsequent um die Fragen nach räumlichen und planerischen Implikationen organisiert ist. $\mathrm{Zu}$ diskutieren wäre, ob mit der Etikettierung als Postwachstumsgeographien nicht eine disziplinäre Engführung vorgenommen wird, über die die Anthologie - insbesondere mit Blick auf planungstheoretische und -praktische Fragestellung - deutlich hinausgeht.

Wie die Herausgeber und Herausgeberin im Vorwort darstellen, ist der rezensierte Band ein Produkt eines laufenden Prozesses zum Thema „Postwachstum“ in der ARL - Akademie für Raumentwicklung in der Leibniz-Gemeinschaft. Es bleibt zu hoffen, dass die hier gestellten Fragen auch zukünftig diskutiert, die Konzepte weiter ausgearbeitet und die vorgeschlagenen Alternativen erprobt werden. Nicht zuletzt aufgrund der Aktualität des Themas, der zahlreichen internationalen Beispiele sowie des Rekurses auf internationale Transformations- und Postwachstumsdebatten, ist es äußerst begrüßenswert, dass dieser aufschlussreiche Band bald auch in englischer Sprache vorliegen wird und somit international rezipiert werden kann.

\section{Vollständige bibliographische Angaben des rezensierten Werkes:}

Lange, Bastian; Hülz, Martina; Schmid, Benedikt; Schulz, Christian (Hrsg.) (2020): Postwachstumsgeographien. Raumbezüge diverser und alternativer Ökonomien. Bielefeld: transcript Verlag. 456 Seiten. 